\title{
In vitro viability effects on apheresis and buffy-coat derived platelets administered through infusion pumps
}

This article was published in the following Dove Press journal:

International Journal of Clinical Transfusion Medicine

3 December 2014

Number of times this article has been viewed

\author{
Per Sandgren ${ }^{1,2}$ \\ Veronica Berggren ${ }^{3}$ \\ Carl Westling ${ }^{1,2}$ \\ Viveka Stiller ${ }^{\prime}$ \\ 'Department of Clinical Immunology \\ and Transfusion Medicine, Karolinska \\ University Hospital, ${ }^{2}$ Department \\ of Laboratory Medicine, Karolinska \\ Institutet, ${ }^{3}$ Department of \\ Neonatology, Karolinska University \\ Hospital, Stockholm, Sweden
}

Background: Different infusion pump systems as well as gravity infusion have been widely used in neonatal transfusion. However, the limited number of published studies describing the use of infusion pumps on platelets illustrates the necessity for more robust data.

Methods: To evaluate the potential in vitro effects on the cellular, metabolic, functional and phenotypic properties of platelets, we set up a four-arm paired study simultaneously comparing the use of different infusion pumps (Alaris ${ }^{\circledR} \mathrm{CC} / \mathrm{GP}$ ) with unexposed platelets. The platelet units $(n=8)$ were either produced by the apheresis technique and suspended in $100 \%$ plasma or derived from buffy coats to yield platelet units stored in approximately $30 \%$ plasma and $70 \%$ SSP+. Fresh and 5-day old platelets were tested.

Results: Regardless of the production system or storage time used, no significant differences were observed in glucose and lactate concentration, $\mathrm{pH}$, adenosine triphosphate levels, response to extent of shape change, hypotonic shock response reactivity, and CD62P expression. Similarly, no differences were observed in expression of the conformational epitope on glycoprotein IIb/ IIIa, determined using procaspase-activating compound 1 , or in the expression of CD42b and platelet-endothelial cell adhesion molecule-1 in a comparison between platelets administered through infusion pumps versus unexposed platelets.

Conclusion: Using Alaris CC/GP infusion pumps had no influence on the cellular, functional, and phenotypic in vitro properties of platelets. This fact seems not to be affected by different production systems or storage time.

Keywords: platelets, neonatal platelet transfusion

\section{Introduction}

Different platelet infusion pump systems ${ }^{1,2}$ as well as gravity infusion have been widely used in neonatal transfusion practice. ${ }^{3}$ Earlier data have shown that these infusion systems do not significantly injure or activate platelets and may also be of clinical benefit to pediatric or adult patients with a history of overload reaction, when precise control of the rate and volume of platelet transfusion is desired. ${ }^{4}$ We note, however, that the favorable effects on platelets have only been evaluated using earlier versions of electromechanical infusion pumps ${ }^{1,2}$ and not using automated pressure-based devices available for this purpose.

One of the key properties of platelets is their capacity to respond rapidly to different types of rheological conditions, become activated and secrete factors that promote blood clotting and tissue regeneration. ${ }^{5}$ Therefore, platelets can be easily triggered when they encounter an artificial environment. Automated or manual pressure-based infusion pumps represent no exception to this notion. The extent to which these
Correspondence: Per Sandgren and Transfusion Medicine, Karolinska University Hospital and Karolinska Institutet, I4I 86 Stockholm, Sweden Fax +46858589410

Email per.sandgren@karolinska.se 
potential manipulations compromise platelet function in vivo is not fully known, but it is nevertheless important to characterize aberrant situations in which platelets capability may be affected. One such potential situation may be that the pressure forces within the infusion pumps, including passage through the pump hoses, further increase factors contributing to existing morphologic, biochemical, or functional derangements caused by processing and storage ${ }^{6-9}$ of the platelet units. Subsequently, such a potential situation may be further associated with an increased risk of decreased post-transfusion survival. ${ }^{7,8,10}$

To evaluate the potential in vitro effects on the cellular, metabolic, functional, and phenotypic properties of platelets, we set up a four-arm paired study comparing the use of different infusion pumps with unexposed platelets. Considering the potential differences in quality of fresh and stored platelets ${ }^{11,12}$ or platelets produced with different methods, ${ }^{13}$ we also investigated the influence of such variables in synergy with the potential effects of using infusion pumps.

\section{Materials and methods Preparation and storage of apheresis platelet units}

Apheresis platelets were collected from healthy blood donors who met standard donation criteria and gave their informed consent according to institutional guidelines. The donors were screened for leukocyte antibodies. The apheresis platelets studied were leukocyte-depleted and suspended in suspended in $100 \%$ plasma. A Trima Accel $^{\circledR}$ automated blood collection system with a single needle (TerumoBCT, Lakewood, CO, USA) was used for the apheresis procedure. ${ }^{14}$ A tubing set with a leukocyte reduction system was used to obtain a product containing $<1 \times 10^{6}$ leukocytes.

The apheresis was performed in a closed system with continuous flow. Whole blood with anticoagulant (ACD-A) was pumped into a separation channel that spins in a centrifuge. The erythrocytes, which are heaviest, end up furthest out, then leukocytes, platelets and the plasma. Platelet-rich plasma was taken to a step-shaped funnel, ie, the leukocyte reduction system chamber, where platelets and leukocytes are separated. The leukocyte-depleted platelet-rich plasma then proceeded through the collection tube to the platelet collection bag (TerumoBCT). The whole procedure took 50-100 minutes. The total extracorporeal volume produced was approximately $196 \mathrm{~mL}$. All units are then stored on a flat-bed agitator in a temperature-controlled cabinet at $22^{\circ} \mathrm{C} \pm 2{ }^{\circ} \mathrm{C}$ (Model PC900i, Helmer, Noblesville, IN, USA). Eight apheresis platelet units were tested on day 2 (fresh) and day 5 (old), respectively. All tested apheresis platelet units were created to fall within clinical requirements.

\section{Preparation and storage of buffy coat platelet units}

Buffy coat platelets were collected according to criteria similar to those for apheresis donation. In total, $450 \mathrm{~mL}$ of whole blood was drawn into either a CPD/SAG-M quadruple-bag blood container system (Fenwal, La Châtre, France) or an NPT 6280LE blood bag system (MacoPharma, Mouvaux, France). After storage at room temperature for 2-6 hours, all whole blood units were centrifuged $(2,700 \mathrm{~g})$ for 10 minutes at $22^{\circ} \mathrm{C}$. Automatic equipment was used for preparation of the blood components (Optipress, Fenwal, or Macopress, Smart, MacoPharma), including buffy coat.

All buffy coat platelet units were prepared using the OrbiSac system (TerumoBCT) ${ }^{15}$ to yield platelet units stored in approximately 30\% plasma and 70\% SSP+ (Macopharma). The buffy coat platelets were stored in platelet storage bags (OrbiSac Standard buffy coat set, storage bag, TerumoBCT Inc.) made of polyvinyl chloride plastic with a citrate-based plasticizer. To avoid disintegration and adverse negative effects on the platelets, ${ }^{16}$ air and foam was excluded from the units immediately after preparation. All units were then stored in the same way as for the apheresis units described earlier (Helmer). Eight buffy coat platelet units were tested on day 2 (fresh) and day 5 (old). All tested buffy coat platelet units were created to fall within clinical requirements.

\section{Infusion pumps and study design}

Alaris ${ }^{\circledR}$ GP volumetric and Alaris CC syringe pumps were used simultaneously from a single apheresis unit or a single buffy coat platelet unit in accordance with the recommendations of the manufacturer (Cardinal Health Inc., Dublin, $\mathrm{OH}, \mathrm{USA}$ ). All reference platelets remained unexposed to the infusion pumps. All samples for in vitro testing were collected in $50 \mathrm{~mL}$ Cellstar tubes (Greiner Bio-one, Monroe, NC, USA). The four-arm paired study is outlined in Figure 1 and was designed as follows:

- A, reference (no run)

- $\mathrm{B}$, smooth aspiration in the syringe, ie, $50 \mathrm{~mL}$ at $90 \mathrm{sec}$ with a Braun Omnifix ${ }^{\circledR} 50 \mathrm{~mL}$ syringe through a BD Connecta ${ }^{\mathrm{TM}}$ three-way stopcock connected to a Mediplast ${ }^{\circledR}$ transfusion unit; the infusion was administered through a BD Connecta extension tube with BD Neoflon ${ }^{\text {TM }} 24 \mathrm{G}(0.7 \times 19 \mathrm{~mm})$ attached to the tube, using the Alaris CC syringe pump 


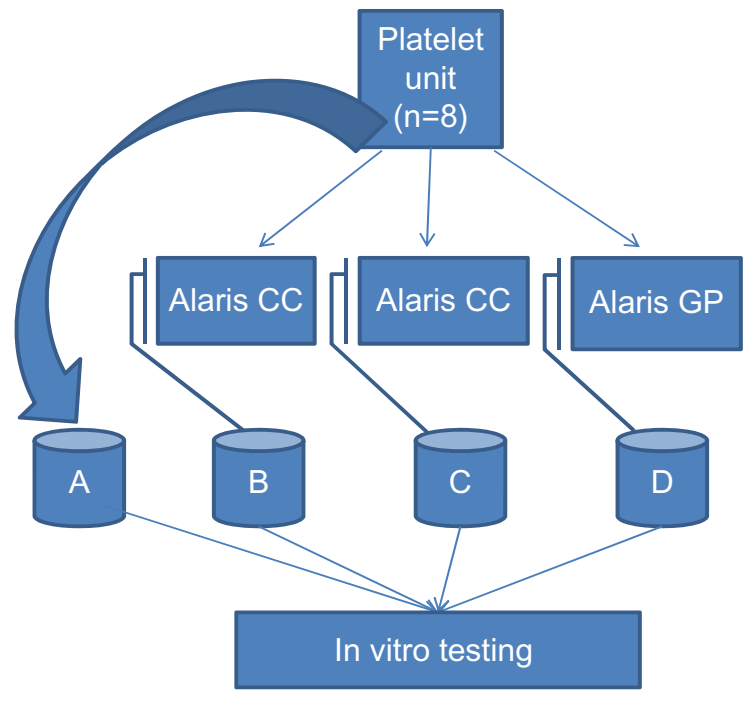

Figure I Schematic overview of the study design.

Note: (B-D) was performed simultaneously from the attached platelet unit with the following pump settings: a total volume of $15 \mathrm{~mL}$ platelet suspension was administered through the different infusion pumps during 30 minutes of total run time while $\mathbf{A}$ remains untreated.

- $\mathrm{C}$, heavy aspiration in the syringe as above but with an aspiration time of less than 10 seconds

- D, infusion was administered using the Alaris GP volumetric pump with the Alaris GP volumetric pump transfusion set, with BD Neoflon $24 \mathrm{G}(0.7 \times 19 \mathrm{~mm})$ connected to the set.

\section{Analysis of cellular, metabolic, in vitro functional, and phenotypic markers}

Immediately after administered through the different infusion pumps or unexposed to infusion pumps, in vitro cellular parameters including measurements of platelet counts $\left(10^{9} / \mathrm{L}\right)$ and mean platelet volume using CA 620 Cellguard (Boule Medical, Stockholm, Sweden) was performed on platelets from the collecting tubes (Greiner Bio-one) (see Figure 1). In the same way, the extracellular metabolic environment was studied using routine blood gas equipment (ABL 800, Radiometer, Copenhagen, Denmark) including $\mathrm{pH}\left(37^{\circ} \mathrm{C}\right), \mathrm{pCO}_{2}$, $\mathrm{pO}_{2}\left(\mathrm{kPa}\right.$ at $\left.37^{\circ} \mathrm{C}\right)$, glucose $(\mathrm{mmol} / \mathrm{L})$, and lactate $(\mathrm{mmol} / \mathrm{L})$. Bicarbonate $(\mathrm{mmol} / \mathrm{L})$ was calculated based on the other measured variables. The $\mathrm{pH}$ of all samples was measured at $37^{\circ} \mathrm{C}$. Therefore, Rosenthal's factor of $0.0147 \mathrm{unit} / 1^{\circ} \mathrm{C}$ was used to correct $\mathrm{pH}$ to the temperature of sampling $\left(22^{\circ} \mathrm{C}\right)$. This factor gives an approximation of the change in $\mathrm{pH}$ of the sample per degree Celsius when it is warmed anaerobically from the collecting temperature of $22^{\circ} \mathrm{C}$ to $37^{\circ} \mathrm{C}$.

According to Bertolini and Murphy, ${ }^{17}$ the assessment of swirling was scored as 0,1 , and 2 . The white blood cell count on day 1 was determined using a Nageotte chamber and a standard microscope (Zeiss, Chester, VA, USA). ${ }^{18}$ Hypotonic shock response reactivity as well as the extent of shape change measurements were performed using a dedicated microprocessor-based instrument (SPA 2000, Chronolog, Havertown, PA, USA) with modifications of these tests as described by VandenBroeke et al. ${ }^{19}$ The total adenosine triphosphate concentration ( $\mu \mathrm{mol} / 10^{11}$ platelets) was determined using a luminometer (Orion Microplate, Berthold Detection Systems GmbH, Pforzheim, Germany) on the basis of the principles described by Lundin. ${ }^{20}$

Extracellular lactate dehydrogenase activity ( $\%$ of total), a marker for cell disintegration, was measured using a spectrophotometric method (kit 063K6003, Sigma-Aldrich, St Louis, MO, USA; 6500 spectrophotometer, Jenway, Stone, UK). ${ }^{21}$ Expression of procaspase-activating compound 1 (a marker of cellular responsiveness towards adenosine diphosphate), CD62P (a marker of activation), CD42b (a marker of adhesive capability), and platelet endothelial cell adhesion molecule 1 (PECAM-1) was measured by flow cytometry (FC500, Beckman Coulter, Villepinte, France). MLP acquisition and analysis software packages (Beckman-Coulter) were used for data acquisition and analysis, respectively. All methods including staining were performed as described in recent publications. ${ }^{16,22,23}$

\section{Statistical analysis}

Mean values and standard deviations are given unless otherwise indicated. A one-way analysis of variance including post hoc test Bonferroni's adjustment was performed. The results of Bonferroni's test are presented in Tables 1-4 and considered to be statistically significant at $P<0.05$. The analyses were carried out using Statistica version 9 software (StatSoft, Inc. 1984-2007 (SPSS, Chicago, IL, USA).

\section{Results}

The platelet counts, cellular, metabolic and in vitro functional and phenotypic parameters are listed and given in Tables 1-4. Initially, we hypothesized that potential differences in the quality of fresh and stored platelets or platelets produced with the different methods might act in synergy with the potential effects of using infusion pumps to further accelerate the aberrant lesion effects on platelets. We note, however, that regardless of production system or storage time, no significant difference in platelet count between the groups was observed by simultaneously using the different infusion pumps. Subsequently, no differences were detected in extracellular lactate dehydrogenase activity in percent of total which remained stable at low levels in both apheresis as 
Table I Cellular, metabolic, functional and phenotypic in vitro effects in fresh apheresis platelets administered through Alaris GP and Alaris CC infusion pumps*

\begin{tabular}{|c|c|c|c|}
\hline & \multicolumn{3}{|c|}{ In vitro marker } \\
\hline & $\begin{array}{l}\text { Platelets } \\
10^{9} / \mathrm{L}\end{array}$ & MPV (fL) & $\begin{array}{l}\text { LDH (\%) } \\
\text { of total }\end{array}$ \\
\hline A Reference (no run) & $1,393 \pm 189$ & $8.6 \pm 1.2$ & $10.5 \pm 4.8$ \\
\hline B Alaris CC (smooth) & $\mathrm{I}, 404 \pm 174$ & $8.6 \pm 1.2$ & $6.6 \pm 4.9$ \\
\hline C Alaris CC (heavy) & $1,395 \pm 190$ & $8.5 \pm 1.1$ & $9.0 \pm 5.4$ \\
\hline \multirow[t]{2}{*}{ D Alaris GP } & $1,394 \pm 198$ & $8.6 \pm 1.2$ & $8.6 \pm 6.3$ \\
\hline & $\begin{array}{l}\text { Glucose } \\
\text { (mmol/L) }\end{array}$ & $\begin{array}{l}\text { Lactate } \\
(\mathrm{mmol} / \mathrm{L})\end{array}$ & $\mathrm{pH}\left(22^{\circ} \mathrm{C}\right)$ \\
\hline A Reference (no run) & $16.7 \pm 7.8$ & $6.6 \pm 2.8$ & $7.509 \pm 0.084$ \\
\hline B Alaris CC (smooth) & $17.0 \pm 8.0$ & $6.7 \pm 2.6$ & $7.579 \pm 0.090$ \\
\hline C Alaris CC (heavy) & $17.0 \pm 8.0$ & $6.7 \pm 2.6$ & $7.597 \pm 0.082$ \\
\hline \multirow[t]{2}{*}{ D Alaris GP } & $17.1 \pm 7.9$ & $6.8 \pm 2.9$ & $7.609 \pm 0.082$ \\
\hline & $\begin{array}{l}\mathrm{pCO}_{2}(\mathrm{kPa} \\
\left.\text { at } 37^{\circ} \mathrm{C}\right)\end{array}$ & Bicarbonate & $\begin{array}{l}\text { ATP }\left(\mu \mathrm{mol} / / 0^{11}\right. \\
\text { platelets) }\end{array}$ \\
\hline A Reference (no run) & $3.88 \pm 0.61$ & $13.5 \pm 2.3$ & $7.9 \pm 0.6$ \\
\hline B Alaris CC (smooth) & $3.22 \pm 0.40^{\dagger}$ & $13.4 \pm 2.4$ & $8.4 \pm 1.0$ \\
\hline C Alaris CC (heavy) & $3.06 \pm 0.32^{\dagger}$ & $13.3 \pm 2.4$ & $8.4 \pm 0.4$ \\
\hline \multirow[t]{2}{*}{ D Alaris GP } & $2.97 \pm 0.37^{\dagger}$ & $13.3 \pm 2.4$ & $9.0 \pm 0.8$ \\
\hline & HSR (\%) & ESC (\%) & CD62P (\%) \\
\hline A Reference (no run) & $61.7 \pm 8.0$ & $25.5 \pm 5.6$ & II.28 23.26 \\
\hline B Alaris CC (smooth) & $54.4 \pm 4.4$ & $24.9 \pm 7.2$ & $11.97 \pm 3.93$ \\
\hline C Alaris CC (heavy) & $56.9 \pm 7.1$ & $25.5 \pm 4.2$ & $|2.8| \pm 3.58$ \\
\hline \multirow[t]{2}{*}{ D Alaris GP } & $60.7 \pm 8.7$ & $28.4 \pm 6.4$ & II. $44 \pm 3.43$ \\
\hline & PAC-I (\%) & CD42b (\%) & PECAM-I (\%) \\
\hline A Reference (no run) & $53.39 \pm 6.58$ & $99.50 \pm 0.15$ & $99.59 \pm 0.35$ \\
\hline B Alaris CC (smooth) & $50.28 \pm 6.78$ & $99.55 \pm 0.12$ & $99.64 \pm 0.38$ \\
\hline C Alaris CC (heavy) & $44.97 \pm 4.94$ & $99.55 \pm 0.15$ & $99.53 \pm 0.56$ \\
\hline \multirow[t]{2}{*}{ D Alaris GP } & $48.37 \pm 5.47$ & $99.50 \pm 0.19$ & $99.63 \pm 0.35$ \\
\hline & $\begin{array}{l}\text { PECAM-I } \\
\text { (MFI) }\end{array}$ & $\begin{array}{l}\mathrm{pO}_{2}(\mathrm{kPa} \\
\left.\text { at } 37^{\circ} \mathrm{C}\right)\end{array}$ & \\
\hline A Reference (no run) & $14.8 \pm 4.9$ & $16.4 \pm 2.7$ & \\
\hline B Alaris CC (smooth) & $14.9 \pm 4.8$ & $20.2 \pm 2 . I^{\dagger}$ & \\
\hline C Alaris CC (heavy) & $14.6 \pm 4.5$ & $21.1 \pm 1.7^{\dagger}$ & \\
\hline D Alaris GP & $14.7 \pm 4.2$ & $20.1 \pm 3.3^{\dagger}$ & \\
\hline
\end{tabular}

Notes: *Values are reported as the mean \pm standard deviation. ${ }^{\dagger} P<0.05$ versus $A$. Abbreviations: ATP, adenosine triphosphate; ESC, extent of shape change; HSR, hypotonic shock response reactivity; LDH, lactate dehydrogenase; MFI, mean fluorescence intensity; MPV, mean platelet volume; PAC-I, procaspase-activating compound I; PECAM-I, platelet endothelial cell adhesion molecule I.

well as buffy coat platelet units. Thus, mean platelet volume remained unaffected when using infusion pumps.

Differences, albeit slight, were found in extracellular $\mathrm{pO}_{2}$ and $\mathrm{pCO}_{2}$ levels $(P<0.05)$, while glucose and lactate concentration, $\mathrm{pH}$, and bicarbonate and adenosine triphosphate levels remained unaffected by the use of the infusion pumps. Subsequently, hypotonic shock response reactivity, the response to extent of shape change, and CD62P expression showed similar responses and expression levels without any significant differences between the units. No differences
Table 2 Cellular, metabolic, functional, and phenotypic in vitro effects in fresh buffy coat-derived platelets administered through Alaris GP and Alaris CC infusion pumps*

\begin{tabular}{|c|c|c|c|}
\hline & \multicolumn{3}{|c|}{ In vitro marker } \\
\hline & $\begin{array}{l}\text { Platelets } \\
10^{9} / \mathrm{L}\end{array}$ & MPV (fl) & $\begin{array}{l}\text { LDH (\%) } \\
\text { of total }\end{array}$ \\
\hline A Reference (no run) & $927 \pm 86$ & $9.0 \pm 0.8$ & $4.7 \pm 1.2$ \\
\hline B Alaris CC (smooth) & $923 \pm 98$ & $8.8 \pm 1.2$ & $4.7 \pm 1.3$ \\
\hline C Alaris CC (heavy) & $926 \pm 90$ & $8.8 \pm 0.8$ & $4.6 \pm 1.9$ \\
\hline \multirow[t]{2}{*}{ D Alaris GP } & $926 \pm 98$ & $8.8 \pm 0.8$ & $3.5 \pm 1.1$ \\
\hline & $\begin{array}{l}\text { Glucose } \\
(\mathrm{mmol} / \mathrm{L})\end{array}$ & $\begin{array}{l}\text { Lactate } \\
(\mathrm{mmol} / \mathrm{L})\end{array}$ & $\mathrm{pH}\left(22^{\circ} \mathrm{C}\right)$ \\
\hline A Reference (no run) & $4.4 \pm 0.7$ & $7.7 \pm 1.2$ & $7.226 \pm 0.071$ \\
\hline B Alaris CC (smooth) & $4.4 \pm 0.7$ & $7.6 \pm 1.0$ & $7.265 \pm 0.068$ \\
\hline C Alaris CC (heavy) & $4.3 \pm 0.7$ & $7.8 \pm 1.0$ & $7.218 \pm 0.039$ \\
\hline \multirow[t]{2}{*}{ D Alaris GP } & $4.4 \pm 0.8$ & $7.8 \pm 1.0$ & $7.230 \pm 0.048$ \\
\hline & $\begin{array}{l}\mathrm{pCO}_{2}(\mathrm{kPa} \\
\left.\text { at } 37^{\circ} \mathrm{C}\right)\end{array}$ & Bicarbonate & $\begin{array}{l}\text { ATP }\left(\mu \mathrm{mol} / 10^{\prime \prime}\right. \\
\text { platelets })\end{array}$ \\
\hline A Reference (no run) & $3.11 \pm 0.61$ & $5.1 \pm 0.8$ & $7.9 \pm 0.9$ \\
\hline B Alaris CC (smooth) & $2.47 \pm 0.5 \mathrm{I}^{\dagger}$ & $4.8 \pm 0.4$ & $8.1 \pm 1.0$ \\
\hline C Alaris CC (heavy) & $2.69 \pm 0.33^{\dagger}$ & $5.1 \pm 0.6$ & $7.9 \pm 1.0$ \\
\hline \multirow[t]{2}{*}{ D Alaris GP } & $2.78 \pm 0.54^{\dagger}$ & $5.0 \pm 0.5$ & $7.6 \pm 0.8$ \\
\hline & HSR (\%) & ESC (\%) & CD62P (\%) \\
\hline A Reference (no run) & $55.0 \pm 9.0$ & $22.1 \pm 4.1$ & $16.68 \pm 2.24$ \\
\hline B Alaris CC (smooth) & $55.2 \pm 5.7$ & $21.7 \pm 3.3$ & $16.41 \pm 3.24$ \\
\hline C Alaris CC (heavy) & $55.6 \pm 5.2$ & $22.9 \pm 2.8$ & $18.72 \pm 2.96$ \\
\hline \multirow[t]{2}{*}{ D Alaris GP } & $56.2 \pm 9.1$ & $23.2 \pm 4.3$ & $|6.37 \pm 2.7|$ \\
\hline & PAC-I (\%) & CD42b (\%) & PECAM-I (\%) \\
\hline A Reference (no run) & $46.98 \pm 5.75$ & $97.62 \pm 2.34$ & $99.48 \pm 0.12$ \\
\hline B Alaris CC (smooth) & $44.78 \pm 7.43$ & $97.56 \pm 2.33$ & $99.53 \pm 0.12$ \\
\hline C Alaris CC (heavy) & $43.68 \pm 7.63$ & $97.19 \pm 1.75$ & $99.42 \pm 0.14$ \\
\hline \multirow[t]{2}{*}{ D Alaris GP } & $44.79 \pm 6.40$ & $97.97 \pm 1.72$ & $99.5 I \pm 0.17$ \\
\hline & $\begin{array}{l}\text { PECAM-I } \\
\text { (MFI) }\end{array}$ & $\begin{array}{l}\mathrm{pO}_{2}(\mathrm{kPa} \\
\left.\text { at } 37^{\circ} \mathrm{C}\right)\end{array}$ & \\
\hline A Reference (no run) & $14.2 \pm 0.9$ & $11.9 \pm 4.2$ & \\
\hline B Alaris CC (smooth) & $14.9 \pm 1.6$ & $13.9 \pm 2.9 \dagger$ & \\
\hline C Alaris CC (heavy) & $14.4 \pm 0.8$ & $13.8 \pm 4.6^{\dagger}$ & \\
\hline D Alaris GP & $15.2 \pm 1.5$ & $14.4 \pm 5.0^{\dagger}$ & \\
\hline
\end{tabular}

Notes: *Values are reported as the mean \pm standard deviation. ${ }^{\dagger} P<0.05$ versus $A$. Abbreviations: ATP, adenosine triphosphate; ESC, extent of shape change; HSR, hypotonic shock response reactivity; LDH, lactate dehydrogenase; MFI, mean fluorescence intensity; MPV, mean platelet volume; PAC-I, procaspase-activating compound I; PECAM-I, platelet endothelial cell adhesion molecule I.

were observed in expression of the conformational epitope on glycoprotein $(\mathrm{Gp}) \mathrm{II} / \mathrm{III}$ a determined by using procaspase-activating compound 1 , as well as in the expression of PECAM-1 and CD42b between groups. Additionally, swirling remained at the highest level $(=2)$ in all tested apheresis and buffy coat platelet units.

\section{Discussion}

This four-armed paired study describes the in vitro viability effects on fresh and stored apheresis and buffy coat platelet 
Table 3 Cellular, metabolic, functional, and phenotypic in vitro effects in stored apheresis platelets administered through Alaris GP and Alaris CC infusion pumps*

\begin{tabular}{|c|c|c|c|}
\hline & \multicolumn{3}{|c|}{ In vitro marker } \\
\hline & $\begin{array}{l}\text { Platelets } \\
10^{9} / \mathrm{L}\end{array}$ & MPV (fl) & $\begin{array}{l}\text { LDH (\%) } \\
\text { of total }\end{array}$ \\
\hline A Reference (no run) & $\mathrm{I}, 327 \pm 20 \mathrm{I}$ & $8.4 \pm 0.7$ & $6.1 \pm 1.0$ \\
\hline B Alaris CC (smooth) & $1,337 \pm 196$ & $8.4 \pm 0.6$ & $6.5 \pm 0.5$ \\
\hline C Alaris CC (heavy) & $1,355 \pm 185$ & $8.4 \pm 0.6$ & $7.2 \pm 1.6$ \\
\hline \multirow[t]{2}{*}{ D Alaris GP } & $1,327 \pm 166$ & $8.3 \pm 0.6$ & $6.8 \pm 1.8$ \\
\hline & $\begin{array}{l}\text { Glucose } \\
(\mathrm{mmol} / \mathrm{L})\end{array}$ & $\begin{array}{l}\text { Lactate } \\
(\mathrm{mmol} / \mathrm{L})\end{array}$ & $\mathrm{pH}\left(22^{\circ} \mathrm{C}\right)$ \\
\hline A Reference (no run) & $11.2 \pm 1.9$ & $12.0 \pm 2.6$ & $7.328 \pm 0.142$ \\
\hline B Alaris CC (smooth) & $11.3 \pm 1.7$ & $11.9 \pm 2.5$ & $7.345 \pm 0.153$ \\
\hline C Alaris CC (heavy) & $1 \mathrm{I} .5 \pm \mathrm{I} .7$ & $11.9 \pm 2.6$ & $7.354 \pm 0.155$ \\
\hline \multirow[t]{2}{*}{ D Alaris GP } & $11.6 \pm 1.5$ & $12.2 \pm 2.8$ & $7.384 \pm 0.156$ \\
\hline & $\begin{array}{l}\mathrm{pCO}_{2}(\mathrm{kPa} \\
\left.\text { at } 37^{\circ} \mathrm{C}\right)\end{array}$ & Bicarbonate & $\begin{array}{l}\text { ATP }\left(\mu \mathrm{mol} / 10^{\prime \prime}\right. \\
\text { platelets })\end{array}$ \\
\hline A Reference (no run) & $3.38 \pm 0.43$ & $7.9 \pm 2.5$ & $7.0 \pm 0.7$ \\
\hline B Alaris CC (smooth) & $2.93 \pm 0.11^{\dagger}$ & $8.0 \pm 2.3$ & $6.9 \pm 1.0$ \\
\hline C Alaris CC (heavy) & $3.13 \pm 0.1$ & $7.9 \pm 2.2$ & $6.6 \pm 1.3$ \\
\hline \multirow[t]{2}{*}{ D Alaris GP } & $2.92 \pm 0.17^{\dagger}$ & $7.8 \pm 2.3$ & $7.0 \pm 1.6$ \\
\hline & HSR (\%) & ESC (\%) & CD62P (\%) \\
\hline A Reference (no run) & $63.2 \pm 2.6$ & $24.0 \pm 3.2$ & $22.08 \pm 4.15$ \\
\hline B Alaris CC (smooth) & $54.6 \pm 3.5$ & $24.2 \pm 3.1$ & $24.03 \pm 4.5$ I \\
\hline C Alaris CC (heavy) & $60.4 \pm 8.2$ & $22.1 \pm 4.4$ & $22.68 \pm 2.87$ \\
\hline \multirow[t]{2}{*}{ D Alaris GP } & $52.6 \pm 7.7$ & $24.4 \pm 1.6$ & $23.26 \pm 6.47$ \\
\hline & PAC-I (\%) & CD42b (\%) & PECAM-I (\%) \\
\hline A Reference (no run) & $27.43 \pm 2.07$ & $99.42 \pm 0.14$ & $99.73 \pm 0.19$ \\
\hline B Alaris CC (smooth) & $27.01 \pm 3.12$ & $99.40 \pm 0.20$ & $99.8 \mathrm{I} \pm 0.04$ \\
\hline C Alaris CC (heavy) & $26.06 \pm 2.31$ & $99.30 \pm 0.27$ & $99.80 \pm 0.04$ \\
\hline \multirow[t]{2}{*}{ D Alaris GP } & $25.27 \pm 1.65$ & $99.37 \pm 0.07$ & $99.77 \pm 0.09$ \\
\hline & $\begin{array}{l}\text { PECAM-I } \\
\text { (MFI) }\end{array}$ & $\begin{array}{l}\mathrm{pO}_{2}(\mathrm{kPa} \\
\left.\text { at } 37^{\circ} \mathrm{C}\right)\end{array}$ & \\
\hline A Reference (no run) & $15.0 \pm 3.3$ & $15.7 \pm 0.2$ & \\
\hline B Alaris CC (smooth) & $15.3 \pm 3.0$ & $17.9 \pm 0.4^{\dagger}$ & \\
\hline C Alaris CC (heavy) & $14.6 \pm 3.2$ & $18.0 \pm 1.2^{\dagger}$ & \\
\hline D Alaris GP & $\mid 5.1 \pm 3.3$ & $18.8 \pm 0.8^{\dagger}$ & \\
\hline
\end{tabular}

Notes: *Values are reported as the mean \pm standard deviation. ${ }^{\dagger} P<0.05$ versus $A$. Abbreviations: ATP, adenosine triphosphate; ESC, extent of shape change; HSR, hypotonic shock response reactivity; LDH, lactate dehydrogenase; MFI, mean fluorescence intensity; MPV, mean platelet volume; PAC-I, procaspase-activating compound I; PECAM-I, platelet endothelial cell adhesion molecule I.

units administered through different infusion pumps as described previously.

With the exception of differences detected in the extracellular environment, all of the in vitro parameters selected on the basis of different aspects of platelet function were not significantly different between the four groups at the measured time points. The data presented demonstrate that platelets administered through infusion pumps maintain their in vitro characteristics to a great extent and do not tend to reinforce potential negative cellular
Table 4 Cellular, metabolic, functional, and phenotypic in vitro effects in stored buffy coat-derived platelets administered through Alaris GP and Alaris CC infusion pumps*

\begin{tabular}{|c|c|c|c|}
\hline & \multicolumn{3}{|c|}{ In vitro marker } \\
\hline & $\begin{array}{l}\text { Platelets } \\
10^{9} / \mathrm{L}\end{array}$ & MPV (fl) & $\begin{array}{l}\text { LDH (\%) } \\
\text { of total }\end{array}$ \\
\hline A Reference (no run) & $863 \pm 151$ & $8.9 \pm 0.3$ & $5.5 \pm 2.4$ \\
\hline B Alaris CC (smooth) & $864 \pm 115$ & $8.9 \pm 0.3$ & $5.9 \pm 2.1$ \\
\hline C Alaris CC (heavy) & $858 \pm 156$ & $8.9 \pm 0.4$ & $3.8 \pm 1.3$ \\
\hline \multirow[t]{2}{*}{ D Alaris GP } & $843 \pm 154$ & $8.8 \pm 0.4$ & $5.2 \pm 2.0$ \\
\hline & $\begin{array}{l}\text { Glucose } \\
(\mathrm{mmol} / \mathrm{L})\end{array}$ & $\begin{array}{l}\text { Lactate } \\
(\mathrm{mmol} / \mathrm{L})\end{array}$ & $\mathrm{pH}\left(22^{\circ} \mathrm{C}\right)$ \\
\hline A Reference (no run) & $3.4 \pm 0.5$ & $9.5 \pm 0.8$ & $7.319 \pm 0.022$ \\
\hline B Alaris CC (smooth) & $3.5 \pm 0.6$ & $9.6 \pm 0.8$ & $7.342 \pm 0.019$ \\
\hline C Alaris CC (heavy) & $3.5 \pm 0.6$ & $9.6 \pm 0.8$ & $7.35 I \pm 0.025$ \\
\hline \multirow[t]{2}{*}{ D Alaris GP } & $3.5 \pm 0.5$ & $9.6 \pm 0.9$ & $7.356 \pm 0.027$ \\
\hline & $\begin{array}{l}\mathrm{pCO}_{2}(\mathrm{kPa} \\
\left.\text { at } 37^{\circ} \mathrm{C}\right)\end{array}$ & Bicarbonate & $\begin{array}{l}\text { ATP }\left(\mu \mathrm{mol} / / 0^{11}\right. \\
\text { platelets })\end{array}$ \\
\hline A Reference (no run) & $2.52 \pm 0.12$ & $5.1 \pm 0.7$ & $7.5 \pm 0.5$ \\
\hline B Alaris CC (smooth) & $2.16 \pm 0.35^{\dagger}$ & $5.0 \pm 0.7$ & $7.5 \pm 0.8$ \\
\hline C Alaris CC (heavy) & $2.09 \pm 0.33^{\dagger}$ & $5.0 \pm 0.6$ & $7.2 \pm 0.4$ \\
\hline \multirow[t]{2}{*}{ D Alaris GP } & $2.03 \pm 0.36^{\dagger}$ & $4.9 \pm 0.7$ & $6.9 \pm 1.1$ \\
\hline & HSR (\%) & ESC (\%) & CD62P (\%) \\
\hline A Reference (no run) & $51.3 \pm 2.9$ & $23.4 \pm 1.0$ & $30.50 \pm 3.52$ \\
\hline B Alaris CC (smooth) & $46.5 \pm 7.3$ & $21.1 \pm 1.6$ & $30.66 \pm 3.53$ \\
\hline C Alaris CC (heavy) & $45.9 \pm 3.8$ & $18.2 \pm 0.6$ & $31.37 \pm 1.74$ \\
\hline \multirow[t]{2}{*}{ D Alaris GP } & $51.4 \pm 3.2$ & $21.8 \pm 3.1$ & $30.34 \pm 4.84$ \\
\hline & PAC-I (\%) & CD42b (\%) & PECAM-I (\%) \\
\hline A Reference (no run) & $32.37 \pm 0.92$ & $95.25 \pm 6.72$ & $99.30 \pm 0.37$ \\
\hline B Alaris CC (smooth) & $29.50 \pm 3.22$ & $94.87 \pm 6.97$ & $99.38 \pm 0.34$ \\
\hline C Alaris CC (heavy) & $30.16 \pm 3.65$ & $95.14 \pm 7.42$ & $99.45 \pm 0.24$ \\
\hline \multirow[t]{2}{*}{ D Alaris GP } & $30.48 \pm 3.29$ & $96.38 \pm 4.60$ & $99.38 \pm 0.36$ \\
\hline & $\begin{array}{l}\text { PECAM-I } \\
\text { (MFI) }\end{array}$ & $\begin{array}{l}\mathrm{pO}_{2}(\mathrm{kPa} \\
\left.\text { at } 37^{\circ} \mathrm{C}\right)\end{array}$ & \\
\hline A Reference (no run) & $17.7 \pm 2.7$ & $11.8 \pm 3.0$ & \\
\hline B Alaris CC (smooth) & $18.3 \pm 1.7$ & $16.5 \pm 2.1^{\dagger}$ & \\
\hline C Alaris CC (heavy) & $17.7 \pm 2.7$ & $17.8 \pm 2.0^{\dagger}$ & \\
\hline D Alaris GP & $18.3 \pm 1.7$ & $18.2 \pm 1.6^{\dagger}$ & \\
\hline
\end{tabular}

Notes: *Values are reported as the mean \pm standard deviation. ${ }^{\dagger} P<0.05$ versus $A$. Abbreviations: ATP, adenosine triphosphate; ESC, extent of shape change; HSR, hypotonic shock response reactivity; LDH, lactate dehydrogenase; MFI, mean fluorescence intensity; MPV, mean platelet volume; PAC-I, procaspase-activating compound I; PECAM-I, platelet endothelial cell adhesion molecule I.

changes, respectively. Hence, the capacity of the different Alaris infusion pump systems (GP/CC) to act as a potential source of morphologic, biochemical, or functional derangements seems to be insignificant. This fact seems not to be affected by the age of the platelets, different production systems used, or heavy aspiration in the syringe preceding use of Alaris CC.

Consequently, a combination of several factors, including different production systems, composition of the platelet storage medium, and biochemical storage effects, needs to 
be considered to conclude that infusion pumps do not affect platelet function.

Extracellular blood gas measurements offer valuable insights into the equilibrium between the cytosolic and mitochondrial oxidative pathways of the platelets, as physiological challenges that disturb metabolic homeostasis cause increased cytosolic glycolysis ${ }^{23-25}$ and promote platelet activation, ${ }^{6,23,26-30}$ as well as aggregation and release reactions. ${ }^{22,23,26,31-34}$ Importantly, the $\mathrm{pH}$ in all the infusion pump-administered fractions was maintained above Council of Europe recommendations ( $\mathrm{pH}>6.4$ ), along with oxygen consumption and the ability to generate an equivalent concentration of adenosine triphosphate, ${ }^{35,36}$ indicating that oxidative phosphorylation was likely maintained in such platelets. The slight observed differences between the groups, including for oxygen and carbon dioxide, may be attributable to differences in the gas exchange capacity of the storage container versus the environmental conditions within the infusion pumps. Because the results for platelets administered through these infusion pumps seem to agree with the functional integrity of the reference platelets, strengthens the conclusion that cellular effects cannot be the cause of the extracellular differences observed.

Platelets have been shown to be variably activated as a consequence of collection, processing, storage, and different pathogen inactivation procedures,,$^{27,37-43}$ although it has been more difficult to directly correlate in vitro platelet activation with the risk of decreased post transfusion recovery and survival. However, it makes sense to consider the consequences of platelet activation as potentially harmful effects $22,31,33,44-48$ and try to minimize and characterize situations in which aberrant platelet activation may occur preceding transfusion.

One such potential situation that caught our attention was aspiration when using the Alaris CC in stressful situations was carried out with vigorous (heavy) aspiration in the syringe, which rationally justifies an additional study arm to examine possible cell influence of this practical procedure. In contrast, offers the volumetric pump no such opportunity to influence the cell content, which eliminates the need for a fifth study arm. In addition, there is great variability worldwide in neonatal platelet transfusion practices ${ }^{49}$ and guidelines differ widely between countries. Therefore, we tested pump settings only according to our local clinical guidelines.

With exception of slight storage effects that were detected for apheresis as well as buffy coat platelets, we observed no statistically verified increase in activation levels in the units tested versus the reference units, which strengthens the conclusion that infusion pumps do not tend to reinforce platelet activation. However, it is important to emphasize that the results presented here are representative of the level of activation of platelets prepared by our specific methods of apheresis and buffy coat platelets, and may not be representative of other devices.

Additionally, using the tested infusion pumps did not affect the ability of the platelets to respond to agonists (extent of shape change and procaspase-activating compound 1), indicating that cellular responsiveness was maintained relative to the activation state. Further, all tested hypotonic shock response reactivity scores were well above the level for which poor in vivo viability has been predicted to occur in a plasma storage environment. ${ }^{50}$

CD42b (Gp1ba) is a subunit of the GpIb-IX-V complex and is the receptor for von Willebrand factor and a high-affinity receptor for thrombin. ${ }^{51}$ A strong correlation between the percentage of platelets able to bind antibodies that recognize the N-terminal of CD42b and adhesive capacity under flow conditions has been reported..$^{52}$ In our study, the percentages of platelets expressing CD42b were all higher $(>90 \%)$ than those associated with decreased adhesive capacity in the abovementioned study. Similarly, we found no difference in the percentage of platelets expressing PECAM-1 (CD31) $)^{53}$ between the groups, and our findings are in accordance with recently presented data on PECAM- 1,54 indicating ultrastructural maintenance and no upregulation of this essential structure.

In summary, our data clearly show that all parameters reflecting different aspects of platelet function remained largely unaffected when administered through Alaris GP and Alaris CC infusion pumps. This observation seems not to be affected by use of different production systems or storage time. However, whether the in vivo efficacy of such platelets is affected will require clinical studies.

\section{Disclosure}

The authors report no conflicts of interest in this work.

\section{References}

1. Norville R, Hinds P, Wilimas J, Fairclough D, Fischl S, Kunkel K. The effects of infusion methods on platelet count, morphology, and corrected count increment in children with cancer: in vitro and in vivo studies. Oncol Nurs Forum. 1994;21(10):1669-1673.

2. Snyder EL, Ferri PM, Smith EO, Ezekowitz MD. Use of an electromechanical infusion pump for transfusion of platelet concentrates. Transfusion. 1984;24:524-527.

3. Murray NA, Roberts IA. Neonatal transfusion practice. Arch Dis Child Fetal Neonatal Ed. 2004 ;89(2):F101-F107. 
4. Snyder EL, Rinder HM, Napychank P. In vitro and in vivo evaluation of platelet transfusions administered through an electromechanical infusion pump. Am J Clin Pathol. 1990;94(1):77-80.

5. Broos K, De Meyer SF, Feys HB, Vanhoorelbeke K, Deckmyn H. Blood platelet biochemistry. Thromb Res. 2012;129(3):245-249.

6. Holme S, Sweeney JD, Sawyer S, Elfath MD. The expression of p-selectin during collection, processing, and storage of platelet concentrates: relationship to loss of in vivo viability. Transfusion. 1997;37(1):12-17.

7. Seghatchian J. Platelet storage lesion: an update on the impact of various leukoreduction processes on the biological response modifiers. Transfus Apher Sci. 2006;34(1):125-130.

8. Seghatchian J, Krailadsiri P. The platelet storage lesion. Transfus Med Rev. 1997;11(2):130-144.

9. Shanwell A, Wikman A, Ringden O. Pretransfusion incubation of apheresis platelets at 37 degrees $\mathrm{C}$ improves posttransfusion recovery. Transfusion. 1992;32(8):715-718.

10. Holme S, Heaton WA, Courtright M. Improved in vivo and in vitro viability of platelet concentrates stored for seven days in a platelet additive solution. Br J Haematol. 1987;66(2):233-238.

11. Diedrich B, Sandgren P, Jansson B, Gulliksson H, Svensson L, Shanwell A. In vitro and in vivo effects of potassium and magnesium on storage up to 7 days of apheresis platelet concentrates in platelet additive solution. Vox Sang. 2008;94(2):96-102.

12. Heuft HG, Goudeva L, Krauter J, Peest D, Buchholz S, Tiede A. Effects of platelet concentrate storage time reduction in patients after blood stem cell transplantation. Vox Sang. 2013;105(1):18-27.

13. Schrezenmeier H, Seifried E. Buffy-coat-derived pooled platelet concentrates and apheresis platelet concentrates: which product type should be preferred? Vox Sang. 2010;99(1):1-15.

14. Bueno JL, Garcia F, Castro E, Barea L, Gonzalez R. A randomized crossover trial comparing three plateletpheresis machines. Transfusion. 2005;45(8):1373-1381.

15. Larsson S, Sandgren P, Sjodin A, Vesterinen M, Gulliksson H Automated preparation of platelet concentrates from pooled buffy coats: in vitro studies and experiences with the OrbiSac system. Transfusion. 2005;45(5):743-751.

16. Sandgren P, Saeed K. Storage of buffy-coat-derived platelets in additive solution: in vitro effects on platelets of the air bubbles and foam included in the final unit. Blood Transfus. 2011;9(2):182-188.

17. Bertolini F, Murphy S. A multicenter evaluation of reproducibility of swirling in platelet concentrates. Biomedical Excellence for Safer Transfusion (BEST) Working Party of the International Society of Blood Transfusion. Transfusion. 1994;34(9):796-801.

18. Moroff G, Eich J, Dabay M. Validation of use of the Nageotte hemocytometer to count low levels of white cells in white cell-reduced platelet components. Transfusion. 1994;34(1):35-38.

19. VandenBroeke T, Dumont LJ, Hunter S, et al. Platelet storage solution effects on the accuracy of laboratory tests for platelet function: a multilaboratory study. Vox Sang. 2004;86(3):183-188.

20. Lundin A. Use of firefly luciferase in ATP-related assays of biomass, enzymes, and metabolites. Methods Enzymol. 2000;305:346-370.

21. Vanderlinde RE. Measurement of total lactate dehydrogenase activity. Ann Clin Lab Sci. 1985;15(1):13-31.

22. Sandgren P, Meinke S, Eckert E, Douagi I, Wikman A, Hoglund P. Random aggregates in newly produced platelet units are associated with platelet activation and release of the immunomodulatory factors sCD40L and RANTES. Transfusion. 2014;54(3):602-612.

23. Sandgren $P$, Stjepanovic A. High-yield platelet units revealed immediate $\mathrm{pH}$ decline and delayed mitochondrial dysfunction during storage in $100 \%$ plasma as compared with storage in SSP+. Vox Sang. 2012;103(1): 55-63.

24. Gulliksson H. Defining the optimal storage conditions for the long-term storage of platelets. Transfus Med Rev. 2003;17(3):209-215.

25. Zhang JG, Carter CJ, Culibrk B, et al. Buffy-coat platelet variables and metabolism during storage in additive solutions or plasma. Transfusion. 2008;48(5):847-856.
26. Apelseth TO, Bruserud O, Wentzel-Larsen T, Bakken AM, Bjorsvik S, Hervig T. In vitro evaluation of metabolic changes and residual platelet responsiveness in photochemical treated and gamma-irradiated singledonor platelet concentrates during long-term storage. Transfusion. 2007;47(4):653-665.

27. Apelseth TO, Hervig T. In vitro evaluation of platelet concentrates during storage: platelet counts and markers of platelet destruction. Transfus Apher Sci. 2007;37(3):261-268.

28. Fijnheer R, Modderman PW, Veldman H, et al. Detection of platelet activation with monoclonal antibodies and flow cytometry. Changes during platelet storage. Transfusion. 1990;30(1):20-25.

29. Fijnheer R, Pietersz RN, de Korte D, et al. Platelet activation during preparation of platelet concentrates: a comparison of the plateletrich plasma and the buffy coat methods. Transfusion. 1990;30(7): 634-638.

30. Rock G, Figueredo A. Metabolic changes during platelet storage. Transfusion. 1976;16(6):571-579.

31. Cognasse F, Boussoulade F, Chavarin P, et al. Release of potential immunomodulatory factors during platelet storage. Transfusion. 2006;46(7):1184-1189.

32. Fullard JF. The role of the platelet glycoprotein IIb/IIIa in thrombosis and haemostasis. Curr Pharm Des. 2004;10(14):1567-1576.

33. Khan SY, Kelher MR, Heal JM, et al. Soluble CD40 ligand accumulates in stored blood components, primes neutrophils through CD40, and is a potential cofactor in the development of transfusion-related acute lung injury. Blood. 2006;108(7):2455-2462.

34. Shattil SJ. Function and regulation of the beta 3 integrins in hemostasis and vascular biology. Thromb Haemost. 1995;74(1):149-155.

35. Holme S, Heaton WA, Courtright M. Platelet storage lesion in secondgeneration containers: correlation with platelet ATP levels. Vox Sang. 1987;53(4):214-220.

36. Kilkson H, Holme S, Murphy S. Platelet metabolism during storage of platelet concentrates at 22 degrees C. Blood. 1984;64(2):406-414.

37. Chavarin P, Cognasse F, Argaud C, et al. In vitro assessment of apheresis and pooled buffy coat platelet components suspended in plasma and SSP+ photochemically treated with amotosalen and UVA for pathogen inactivation (INTERCEPT blood system). Vox Sang. 2011;100(2):247-249.

38. Goodrich RP, Edrich RA, Li J, Seghatchian J. The Mirasol PRT system for pathogen reduction of platelets and plasma: an overview of current status and future trends. Transfus Apher Sci. 2006;35(1): $5-17$.

39. Lin L, Dikeman R, Molini B, et al. Photochemical treatment of platelet concentrates with amotosalen and long-wavelength ultraviolet light inactivates a broad spectrum of pathogenic bacteria. Transfusion. 2004;44(10):1496-1504.

40. Sandgren P, Callaert M, Shanwell A, Gulliksson H. Storage of platelet concentrates from pooled buffy coats made of fresh and overnight-stored whole blood processed on the novel Atreus $2 \mathrm{C}+$ system: in vitro study. Transfusion. 2008;48(4):688-696.

41. Sandgren P, Hild M, Sjodin A, Gulliksson H. Storage of buffy-coatderived platelets in additive solutions: in vitro effects on platelets prepared by the novel TACSI system and stored in plastic containers with different gas permeability. Vox Sang. 2010;99(4):341-347.

42. Sandgren P, Tolksdorf F, Struff WG, Gulliksson H. In vitro effects on platelets irradiated with short-wave ultraviolet light without any additional photoactive reagent using the THERAFLEX UV-platelets method. Vox Sang. 2011;101(1):35-43.

43. Sandgren P, van Waeg G, Verheggen C, Sjodin A, Gulliksson H. Storage of interim platelet units for 18 to 24 hours before pooling: in vitro study. Transfusion. 2011;51(6):1213-1219.

44. Apelseth TO, Hervig TA, Wentzel-Larsen T, Bruserud O. Cytokine accumulation in photochemically treated and gamma-irradiated platelet concentrates during storage. Transfusion. 2006;46(5):800-810.

45. Chicheportiche Y, Bourdon PR, Xu H, et al. TWEAK, a new secreted ligand in the tumor necrosis factor family that weakly induces apoptosis. J Biol Chem. 1997;272(51):32401-32410. 
46. Cognasse F, Osselaer JC, Payrat JM, Chavarin P, Corash L, Garraud O. Release of immune modulation factors from platelet concentrates during storage after photochemical pathogen inactivation treatment. Transfusion. 2008;48(5):809-813.

47. Iuliano L, Colavita AR, Leo R, Pratico D, Violi F. Oxygen free radicals and platelet activation. Free Radic Biol Med. 1997;22(6): 999-1006.

48. Leo R, Pratico D, Iuliano L, et al. Platelet activation by superoxide anion and hydroxyl radicals intrinsically generated by platelets that had undergone anoxia and then reoxygenated. Circulation. 1997;95(4): 885-891.

49. Christensen RD. Advances and controversies in neonatal ICU platelet transfusion practice. Adv Pediatr. 2002;55:255-269.
50. Holme S. Storage and quality assessment of platelets. Vox Sang. 1998; 74 Suppl 2:207-216.

51. Berndt MC, Shen Y, Dopheide SM, Gardiner EE, Andrews RK. The vascular biology of the glycoprotein Ib-IX-V complex. Thromb Haemost. 2001;86(1):178-188.

52. Henrita van Zanten G, Saelman EU, Schut-Hese KM, et al. Platelet adhesion to collagen type IV under flow conditions. Blood. 1996;88(10): 3862-3671.

53. Falati S, Patil S, Gross PL, et al. Platelet PECAM-1 inhibits thrombus formation in vivo. Blood. 2006;107(2):535-541.

54. Sandgren P, Larsson S, Wai-San P, Aspevall-Diedrich B. The effects of pneumatic tube transport on fresh and stored platelets in additive solution. Blood Transfus. 2014;12(1):85-90.

\section{Publish your work in this journal}

International Journal of Clinical Transfusion Medicine is an international, peer-reviewed, open access, online journal publishing clinicalexperimental, policy-making and evidence-based practices of all topics pertaining to clinical transfusion medicine. Original research, short reports, reviews, case reports and commentaries are invited.
The manuscript management system is completely online and includes a very quick and fair peer-review system, which is all easy to use. Visit http://www.dovepress.com/testimonials.php to read real quotes from published authors. 